\title{
A partial response to abatacept in a patient with steroid resistant focal segmental glomerulosclerosis
}

\author{
Eren Soyaltın ${ }^{1 \oplus}$, Belde Kasap Demir ${ }^{1,2 \odot}$, Caner Alparslan ${ }^{1 \oplus}$, \\ Seçil Arslansoyu Çamlar ${ }^{1 \oplus}$, Demet Alaygut ${ }^{1 \oplus}$, Önder Yavaşcan ${ }^{3 \oplus}$, Fatma Mutlubaş ${ }^{1 \oplus}$ \\ ${ }^{1}$ Department of Pediatric Nephrology, University of Health Sciences, Tepecik Training and Research Hospital, ${ }^{2}$ Department of Pediatric \\ Nephrology, Katip Çelebi University Faculty of Medicine, Izmir; ${ }^{3}$ Department of Pediatric Nephrology, İstanbul Medipol University \\ Faculty of Medicine, İstanbul Turkey.
}

\begin{abstract}
Background. Herein we present our experience with abatacept in a patient diagnosed with primary focal segmental glomerulosclerosis (FSGS) and resistant to steroid and other immunosuppressives.

Case. A 17-year-old girl was diagnosed with idiopathic nephrotic syndrome (NS) at the age of 8 years. Kidney biopsy was performed when she did not respond to 6-weeks of steroid $(2 \mathrm{mg} / \mathrm{kg})$ therapy followed by three doses of pulse methylprednisolone (PMP) and considered as steroid resistant NS. The biopsy revealed focal segmental glomerulosclerosis (FSGS) and cyclophosphamide was added to the steroid treatment but the patient had no response. The genetic analysis revealed G34G/A318A compound homozygous synonym aminoacid variation in NPHS2 gene, thus all immunosuppressive regimes were stopped and she was put on supportive treatment. Throughout this period, she had nephrotic range of proteinuria, however serum albumin levels were $>3 \mathrm{~g} / \mathrm{dl}$. At the end of two years, the patient had NS with severe edema and hypoalbuminemia. When the genetic analysis was interpreted again, it was found to be consistent with a polymorphism rather than a mutation. Following 3 doses of PMP, oral steroid treatment was resumed and cyclosporine (CsA) was added to the treatment at the fifth year of follow up. However, she was unresponsive to CsA at the end of the first year as well as mycophenolate mofetil used for 12 months and rituximab used for 6 months, respectively. Then abatacept was instituted and proteinuria decreased below $1 \mathrm{gr} /$ day and serum albumin levels increased to $3 \mathrm{~g} / \mathrm{dl}$ at the end of 6 doses. Serum albumin levels remained stable in the following 7 months.

Conclusion. Partial remission including the decrease in proteinuria and increase in albumin levels achieved in our patient encourages the usage of abatacept in patients who do not respond to multiple immunosuppressive therapies.
\end{abstract}

Key words: focal segmental glomerulosclerosis, abatacept, nephrotic syndrome.

Idiopathic nephrotic syndrome (INS) is the most common glomerular disease in childhood. ${ }^{1,2}$ It is classified according to the response to steroid treatment as steroid-sensitive nephrotic syndrome (SSNS) or steroid-resistant nephrotic syndrome (SRNS). ${ }^{3}$ Although most children are diagnosed with SSNS, 10-20\% of patients have no response to steroids and are diagnosed with SRNS. These patients

$\triangle$ Eren Soyaltın

erensoyaltin@hotmail.com

Received 11th July 2019, revised 30th December 2019, 26th October 2019, accepted 31st January 2020. have a greater risk for end-stage renal failure when compared to patients with SSNS. ${ }^{4}$ The etiology of SRNS is also unknown in children; however podocytopathies are considered to be responsible for both minimal change disease (MCD) and focal segmental glomerulosclerosis (FSGS). The circulating factors derived from immune dysregulation may also cause podocyte injury as well as genetic structural defect. ${ }^{5,6}$

Steroid resistance is defined as a failure to response to 4 weeks of daily therapy and high-dose steroid pulses. ${ }^{1,7}$ Following failure of standard steroid therapy, second-line 
immunosuppressive agents like calcineurin inhibitors, cyclophosphamide (CYC), mycophenolate mofetil (MMF), Rituximab (RTX); can be initiated in combination with corticosteroids for remission. ${ }^{3,7}$ A new biological agent abatacept, a cytotoxic T-lymphocyteassociated antigen 4-immunoglobulin fusion protein [CTLA-4-Ig] is available in recent studies. ${ }^{5,8}$ Herein we present our experience with Abatacept in a patient diagnosed with primary FSGS and resistant to steroid and second-line immunosuppressive agents.

\section{Case Report}

A 17-year-old girl, diagnosed with INS at the age of 8 years, had been treated with oral steroids $(2 \mathrm{mg} / \mathrm{kg} /$ day $)$ as initial treatment. When she did not respond to 6-weeks of oral steroid therapy followed by three doses of daily pulse methylprednisolone (PMP), she was considered as SRNS and a kidney biopsy was performed, which confirmed FSGS as the underlying pathology. Cyclophosphamide (1 $\mathrm{mg} / \mathrm{kg} /$ day orally) was added to the steroid treatment. The genetic analysis revealed G34G/ A318A compound homozygous synonym amino acid variation in NPHS-2 gene. Then, immunosuppressive therapies were discontinued; angiotensin converting enzyme inhibitor (ACEI), angiotensin receptor blocker (ARB), fish oil and statin treatment were initiated. Throughout this two-year period, she had nephrotic range of proteinuria, however serum albumin levels were $>3 \mathrm{~g} / \mathrm{dl}$. At the end of two years, the patient had NS with severe edema and hypoalbuminemia. When the genetic analysis was interpreted again, it was found to be consistent with a polymorphism rather than a mutation. Further genetic analysis revealed no mutation in NPHS-1, NPHS-3, NPHS6, TRPC6, WT1, LAMB2, DGKE, ARHGDIA, COQ2, COQ6, CD2AP, ACTN4, CRB2, INF2, PAX2, MYO1E, APOL1 and ADCK-4 genes either. Following 3 doses of daily PMP, oral steroid treatment was resumed. In the follow-up, partial remission (50\% decrease in proteinuria and serum albumin $>3 \mathrm{~g} / \mathrm{dl}$ ) was achieved and cyclosporine (CsA) $(5 \mathrm{mg} / \mathrm{kg} /$ day) was added to the treatment. When she experienced relapses in the first year of CsA treatment, RTX (375 $\mathrm{mg} / \mathrm{m}^{2} /$ dose) was added to her therapy and CD19 levels were measured weekly. Second dose of RTX was given one month after the first dose due to increased CD19 levels (>1\%). The administration of second dose of RTX was associated with a dramatic fall in urinary protein excretion $\left(120 \mathrm{mg} / \mathrm{m}^{2} /\right.$ hour to $40.5 \mathrm{mg} /$ $\mathrm{m}^{2}$ /hour). At the sixth month of therapy, a third dose of RTX was given due to increased levels of CD19; but this time she did not respond to therapy well. Then, steroid and CsA therapies were stopped by tapering doses. Supportive treatments including ACEI and ARB were sustained and MMF $\left(500 \mathrm{mg} / \mathrm{m}^{2} /\right.$ dose at 2 doses) was initiated. However, there was no response to MMF treatment. She had severe proteinuria with albumin levels between 2 and $2.5 \mathrm{~g} / \mathrm{dl}$ causing edema. At the end of 1.5 years, MMF therapy was discontinued and abatacept was initiated. She received six doses of abatacept (750 mg/dose) in 4 months as three doses over a month, then at monthly intervals. After administration of third dose of abatacept; the patient achieved partial remission and albumin levels increased above $3 \mathrm{~g} / \mathrm{dl}$ (Fig. 1). As planned, 6 doses of abatacept were applied without any adverse effects and the proteinuria level decreased to $40 \mathrm{mg} / \mathrm{m}^{2} /$ hour after the last dose. One month after the last dose, the patient had $160 \mathrm{mg} / \mathrm{m}^{2} /$ hour of proteinuria, however albumin levels were over $3 \mathrm{~g} / \mathrm{dl}$ and she had no edema anymore. At her last visit 7 months after the last dose abatacept, 24-hour proteinuria was $120 \mathrm{mg} / \mathrm{m}^{2} /$ hour, but serum albumin was $3.1 \mathrm{~g} / \mathrm{dl}$, serum creatinine was $0.6 \mathrm{mg} / \mathrm{dl}$ and the glomerular filtration rate was $142 \mathrm{~mL} / \mathrm{min}$ per $1.73 \mathrm{~m}^{2}$. There was no decrease in mean glomerular filtration rate values compared to pretreatment period $\left(131.5 \mathrm{~mL} / \mathrm{min}\right.$ per $\left.1.73 \mathrm{~m}^{2}\right)$.

An informed consent was received from the family to report the patient. 


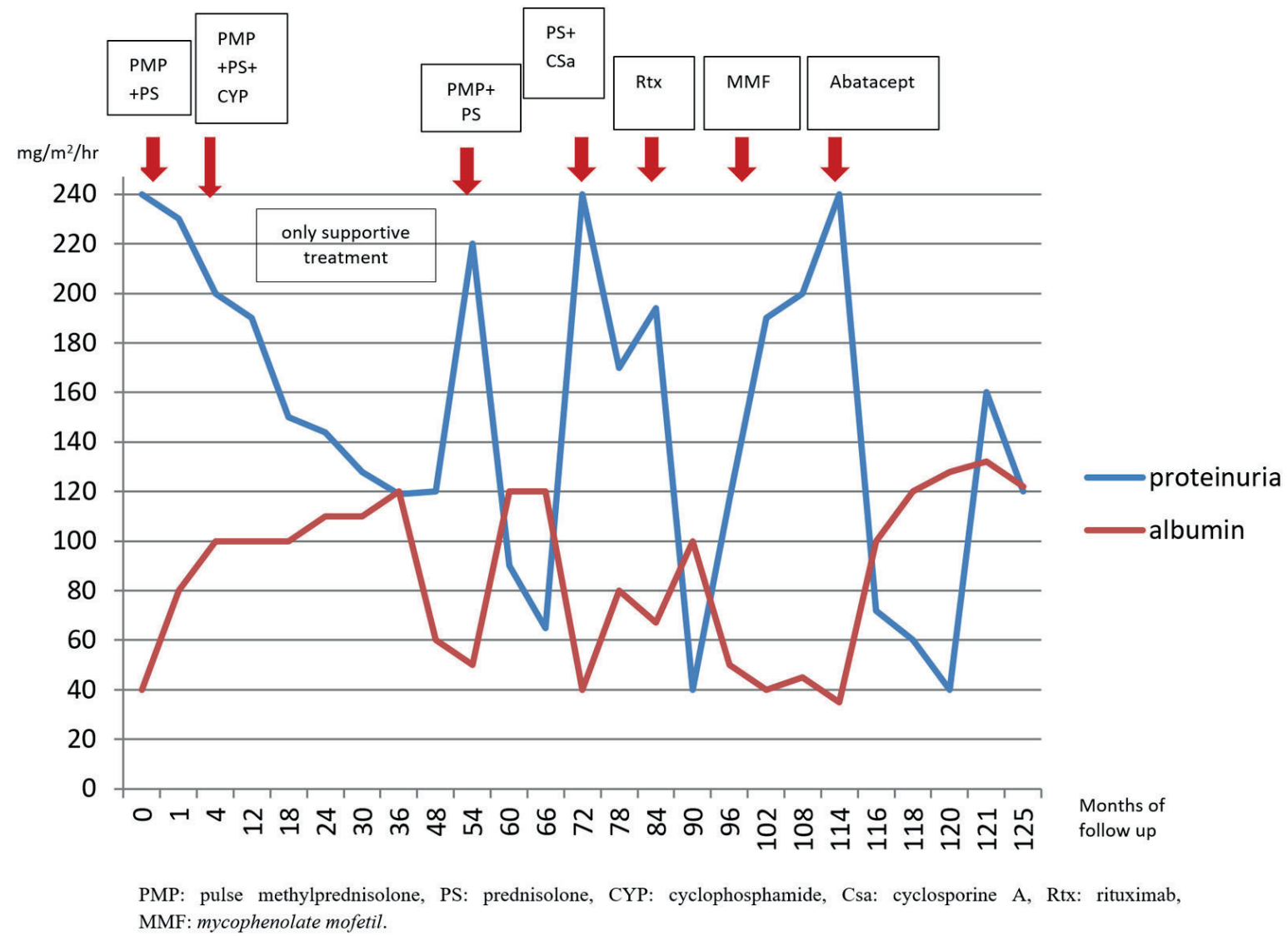

Fig. 1. The proteinuria, albumin levels and the treatments of the patient in follow up period.

\section{Discussion}

FSGS is a histological variant of INS and is considered as a podocytopathy. In recent studies, the expression of de novo CD80 in podocytes has been reported during relapse period and it is suggested to be a causal role for podocyte injury and proteinuria by disrupting the binding of talin to $B 1$ integrin. ${ }^{3,5,9}$ CTLA-4 is a CD80 inhibitor and is expressed in human podocytes. ${ }^{5,10}$ Abatacept is a cytotoxic T-lymphocyte-associated antigen 4-immunoglobulin fusion protein and a costimulatory B7-1 (CD80) inhibitor. ${ }^{5,8,11} \mathrm{Yu}$ et al. ${ }^{8}$ reported partial or complete remission with abatacept in one patient with glucocorticoidresistant primary FSGS and four patients with RTX-resistant recurrent FSGS after kidney transplantation (KTx). B7-1 staining of podocytes was shown in the biopsy specimens of whole patients and it has been shown to be a predictive factor for responding to abatacept therapy. Alachkar et al. ${ }^{12}$ shared data with respect to five patients with FSGS after KTx who did not have a response to plasmapheresis and RTX. The patients did not have any positive therapeutic response to abatacept treatment; despite positive podocyte B7-1 expression in their kidney-biopsy specimens. Delvillle et al. ${ }^{13}$ reported a prospective study of 9 patients with recurrent FSGS after KTx, with no remission after abatacept therapy in contrast with the recent report by $\mathrm{Yu}$ et al. ${ }^{8}$ B7-1 expression was not detected in podocytes of biopsies performed at the time of recurrence. Following this study, Delvielle et al. ${ }^{13}$ tested B7-1 in other proteinuric diseases of native kidneys including primary and secondary forms of FSGS, diabetic nephropathy, membranous nephropathy, 
ANCA vasculitis, and lupus nephritis and B7-1 was not detectable in podocytes in any of these patients. As a result, they did not support the use of B7-1 immunostaining as a biomarker to treat patients with abatacept. Novelli et al. ${ }^{14}$ analyzed B7-1 protein expression in the kidney biopsies of 31 patients diagnosed with MCD and FSGS and they could not show B7-1 expression on podocytes in biopsy specimens. They suggest that the anti-proteinuric effect of abatacept determined in previous studies may be a result of immune cell inhibiting rather than a direct effect on podocytes.

The necessity of B7-1 staining in biopsy preparations before initiation of treatment still contains a suspicion and its relationship with disease activation and treatment has not been clearly demonstrated yet. In our case, we thought that we would start abatacept treatment despite any evidence for B7-1 staining due to this uncertainty. In addition, B7-1 immunstaining was not available in our pathology clinic.

Jayaraman et al. ${ }^{15}$ reported abatacept experience in a 62 year-old man with steroid and RTX resistant FSGS, who failed to respond to therapy. In addition, Garin et $a 1 .{ }^{5}$ have reported progressive fall in urinary protein excretion after abatacept administration in a patient with MCD and high urinary CD80 excretion, in contrast to patients diagnosed with FSGS (one patient with primary FSGS and three patients with recurrent FSGS after KTx) and no CD80 excretion. However, they reported that proteinuria increased on day 9 in their patient with MCD, who responded to abatacept. In our patient, proteinuria decreased to non-nephrotic ranges and albumin levels increased following the third dose of abatacept; but as stated by Garin et al. ${ }^{5}$, proteinuria increased rapidly 1 month after the last dose of abatacept.

In our report, there appears to be a similarity between the response of Abatacept and RTX. Partial remission was achieved after RTX treatment and once after pulse steroid administration too. However, with these treatments, the response was not long-lasting, was not achieved at repeated doses and required additional immunosuppressive treatments. There are different protocols of RTX applied in NS but there is still no certain protocol yet. One of these protocols is to administer the drug, once weekly for four weeks. ${ }^{16}$ We prefered to follow the patients with serum CD19 levels considering the possible side effects of RTX and repeat the doses when CD19 levels increase above $1 \%$. Although we received a partial response after the second dose, we did not see any benefit after the last dose. Although partial remission was achieved only once with pulse steroid treatment, relapse was observed rapidly on the follow-up period. On the other hand, we did not prefer repeated applications due to side effects such as cataract and impaired glucose tolerance.

In conclusion, although the hope for abatacept failed with regard to the studies carried out after the promising publications of $\mathrm{Yu}$ et al. ${ }^{8}$ and Alackhar et al. ${ }^{12}$ because of the negative consequences of the anti-proteinuric effect of the drug, partial remission including the decrease in proteinuria and increase in albumin levels achieved in our patient encourages the usage of abatacept in patients who do not respond to multiple immunosuppressive therapies and decreased quality of life due to persistent edema and recurrent hospitalizations. As there is no consensus in terms of duration, dosages, safety and efficiency of abatacept yet, further studies to clarify these issues are needed.

\section{REFERENCES}

1. Bensimhon AR, Williams AE, Gbadegesin RA. Treatment of steroid-resistant nephrotic syndrome in the genomic era. Pediatr Nephrol 2019; 34: 22792293.

2. Eddy AA, Symons JM. Nephrotic syndrome in childhood. Lancet 2003; 362: 629-639.

3. Kemper MJ, Valentin L, van Husen M. Difficult-totreat idiopathic nephrotic syndrome: established drugs, open questions and future options. Pediatr Nephrol 2018; 33: 1641-1649. 
4. Jellouli M, Charfi R, Maalej B, Mahfoud A, Trabelsi S, Gargah T. Rituximab in the management of pediatric steroid-resistant nephrotic syndrome: a systematic review. J Pediatr 2018; 197: 191-197.e1.

5. Garin EH, Reiser J, Cara-Fuentes G, et al. Case series: CTLA4-IgG1 therapy in minimal change disease and focal segmental glomerulosclerosis. Pediatr Nephrol 2015; 30: 469-477.

6. Barisoni L, Schnaper HW, Kopp JB. A proposed taxonomy for the podocytopathies: a reassessment of the primary nephrotic diseases. Clin J Am Soc Nephrol 2007; 2: 529-542.

7. Primary nephrotic syndrome in children: clinical significance of histopathologic variants of minimal change and of diffuse mesangial hypercellularity. A report of the International Study of Kidney Disease in Children. Kidney Int 1981; 20: 765-771.

8. Yu CC, Fornoni A, Weins A, et al. Abatacept in B71-positive proteinuric kidney disease. N Engl J Med 2013; 369: 2416-2423.

9. Reiser J, von Gersdorff G, Loos M, et al. Induction of B7-1 in podocytes is associated with nephrotic syndrome. J Clin Invest 2004; 113: 1390-1397.

10. Ishimoto T, Shimada M, Gabriela G, et al. Toll-like receptor 3 ligand, polyIC, induces proteinuria and glomerular CD80, and increases urinary CD80 in mice. Nephrol Dial Transplant 2013; 28: 1439-1446.
11. Liu Y, Shi Y, Ren R, Xie J, Wang W, Chen N. Advanced therapeutics in focal and segmental glomerulosclerosis. Nephrology (Carlton). 2018; 23(Suppl 4): 57-61.

12. Alachkar N, Carter-Monroe N, Reiser J. Abatacept in B7-1-positive proteinuric kidney disease. N Engl J Med 2014; 370: 1263-1264.

13. Delville M, Baye E, Durrbach A, et al. B7-1 blockade does not improve post-transplant nephrotic syndrome caused by recurrent FSGS. J Am Soc Nephrol 2016; 27: 2520-2527.

14. Novelli R, Gagliardini E, Ruggiero B, Benigni A, Remuzzi G. Any value of podocyte B7-1 as a biomarker in human MCD and FSGS? Am J Physiol Renal Physiol 2016; 310: F335-F341.

15. Jayaraman VK, Thomas M. Abatacept experience in steroid and rituximab-resistant focal segmental glomerulosclerosis. BMJ Case Rep 2016; 2016: 214396.

16. Kamei K, Ishikura K, Sako M, et al; Rituximab for Childhood-Onset Refractory Nephrotic Syndrome (RCRNS) Study Group. Long-term outcome of childhood-onset complicated nephrotic syndrome after a multicenter, double-blind, randomized, placebo-controlled trial of rituximab. Pediatr Nephrol 2017; 32: 2071-2078. 